\title{
Estudo comparativo entre cuidadores formais e informais de idosos
}

\author{
Comparative study between formal and informal caregivers \\ of older adults
}

\author{
Maria Angélica Andreotti Diniz ${ }^{1}$ \\ Beatriz Rodrigues de Souza Melo ${ }^{1}$ \\ Karolina Helena Neri ${ }^{2}$ \\ Francine Golghetto Casemiro ${ }^{1}$ \\ Leandro Correa Figueiredo ${ }^{3}$ \\ Cheila Cristina Leonardo de Oliveira Gaioli ${ }^{4}$ \\ Aline Cristina Martins Gratão ${ }^{2}$
}

\footnotetext{
${ }^{1}$ Programa de Pós-

Graduação em Enfermagem,

Universidade Federal de

São Carlos (UFSCar). Rod.

Washington Luis km 235.

13565-905 São Carlos

SP Brasil.

maah-diniz@hotmail.com

${ }^{2}$ Departamento de

Gerontologia, UFSCar. São

Carlos SP Brasil.

${ }^{3}$ Programa de Pós-

Graduação em Fisioterapia,

UFSCar. São Carlos SP

Brasil.

${ }^{4}$ Pós-Graduação em

Enfermagem Psiquiátrica,

Escola de Enfermagem de

Ribeirão Preto, Universidade

de São Paulo. Ribeirão Preto

SP Brasil.
}

\begin{abstract}
This study was aimed to compare the health conditions and burden of formal and informal caregivers of older adults. Cross-sectional and comparative study with a quantitative approach. The data were collected in 2014 in São Carlos/SP, using the Zarit Burden Interview and the SRQ 20 (Self Reported Questionnaire). Results: The sample included 15 formal and 35 informal caregivers. Among the formal caregivers, women were predominant (86.7\%), with a mean age of 36.7 , mean of 13.7 years of education, mean workload of 7.5 hours per day, 26.7\% were diagnosed with emotional distress and they the majority mentioned feeling "a little overloaded" (40\%). Concerning the informal caregivers, women were predominant $(85.7 \%), 42.9 \%$ were the children of the older adult, with a mean age of 55.2 years, mean of 7.1 years of education, length of time as caregiver of 6.5 years, mean of 19.8 hours per day taking care of the older adult, 17 (48.6\%) presented mild overload and 16 (45.7\%) presented emotional distress. Conclusions: The results revealed important differences and attention is needed to planning interventions to improve health

Resumo Este estudo objetivou comparar as condições de saúde e a sobrecarga de cuidadores formais e informais de idosos. Estudo observacional, seccional, comparativo com coleta em 2014, em São Carlos/SP, utilizando-se os instrumentos Escala de Sobrecarga de Zarit e SRQ 20 (Self Reporting Questionaire). A amostra, constituida de 15 cuidadores formais e 35 informais. Os formais, maioria mulheres (86,7\%), média de 36,7 anos, escolaridade de 13,7 anos, carga horária de 7,5 horas diárias de trabalho, 26,7\% com desconforto emocional e maior frequência, sentiram-se "um pouco sobrecarregados" (40\%). Os informais, maioria mulheres (85,7\%), 42,9\% representados por filhos, média de 55,2 anos, escolaridade de 7,1 anos, tempo de cuidado de 6,5 anos, com 19,8 horas diárias no cuidado ao idoso, 17 (48,6\%) apresentaram leve sobrecarga e 16 (45,7\%) apresentaram desconforto emocional. Revelaram-se importantes diferenças e alertas para o planejamento de intervenções visando à melhoria nas condições de saúde e de trabalho destes indivíduos. Palavras-chave Estresse psicológico, Cuidadores, Idosos
\end{abstract} and work conditions of these people.

Key words Psychological stress, Caregivers, Older adults 


\section{Introdução}

Os resultados da última Pesquisa Nacional por Amostra de Domicílios (PNAD) revelaram que, no Brasil, o número de idosos com 60 anos ou mais de idade passou de 21,7 milhões, em 2009; e para 27,8 milhões, em 2014. Entre os idosos longevos, o aumento é ainda maior. Em 2009, o Brasil registrava 9,7 milhões de pessoas com mais de 70 anos, enquanto, em 2014, a população dessa faixa etária atingiu um efetivo de 12,2 milhões de idosos ${ }^{1}$.

Com esse panorama de crescimento populacional, é notável uma alteração do perfil epidemiológico da população, com alta prevalência das doenças crônicas não transmissíveis, perdas cognitivas, declínio sensorial, acidentes e isolamento social. O que causam perdas da capacidade funcional nos idosos tornando-os dependentes de outros para a prestação dos cuidados diários ${ }^{2}$.

Diante desse contexto, a tarefa do cuidar, representada pelo papel do cuidador, inclui ações que visam auxiliar o idoso impedido física ou mentalmente a desempenhar tarefas práticas das atividades da vida diária e autocuidado. Definese cuidador, aquele que é responsável por cuidar da pessoa doente ou dependente, facilitando o exercício de suas atividades diárias, tais como alimentação, higiene pessoal, oferecimento da medicação de rotina e acompanhamento aos serviços de saúde, ou outros requeridos no seu cotidiano ${ }^{3}$.

Com a tarefa de cuidar do idoso, geralmente, de caráter ininterrupto, em atividades como cuidados corporais, alimentação, eliminações, ambiente, oferecimento de medicações e outras situações, o cuidador pode experimentar situações desgastantes e de sobrecarga ${ }^{4}$.

Devido às demandas desgastantes que os cuidadores e familiares passam com o cuidado ao idoso, é importante avaliar e minimizar a sobrecarga do profissional responsável pelo cuidado ${ }^{5}$.

As sobrecargas de atividades geradas aos cuidadores podem levar ao desenvolvimento de sintomas psiquiátricos e inclusive necessidade do uso de medicamentos psicotrópicos, o que pode comprometer a capacidade do cuidador para cuidar do idoso. A atividade de cuidar pode ter efeitos sociais e econômicos que comprometem todos os aspectos da vida das pessoas envolvidas ${ }^{6}$.

Independente do cuidado ser formal ou informal, esses cuidadores carecem de suporte profissional e de um espaço para compartilhamento de dúvidas e anseios.A atenção integral à saúde do cuidador deve ser baseada na habilidade em estabelecer as necessidades de saúde dessa população e de programar e avaliar as intervenções sanitárias relativas ao cuidado para as pessoas individualmente em cada contexto.

Nessa perspectiva, o presente estudo buscou avaliar as condições de saúde, nível de sobrecarga e desconforto emocional dos cuidadores formais e informais dos idosos que passaram por atendimento em três diferentes níveis de complexidade: hospitalar, ambulatorial e instituição de longa permanência, favorecendo assim, o aprimoramento do planejamento de ações integrais em saúde para essas pessoas.

\section{Método}

Trata-se de um estudo observacional, seccional, comparativo e com abordagem quantitativa para verificação dos dados sóciodemográficos, de saúde e do nível de sobrecarga e de desconforto emocional dos cuidadores de idosos.

Os participantes foram recrutados em 2 grupos. No primeiro, os cuidadores informais foram identificados no atendimento médico ao idoso na Unidade Saúde Escola (USE), considerada um ambulatório de média complexidade que atende usuários referenciados pela rede pública de saúde de São Carlos e região. Além desse local, foram recrutados cuidadores informais de idosos atendidos no Hospital Universitário da UFSCar, que está integrado à Rede (Unidades de Atenção Básica e Secundária) oferecendo atenção à saúde prestando serviços de observação/internação e atendimentos de urgência à população. $\mathrm{O}$ segundo grupo foi representado por cuidadores formais de uma Instituição Filantrópica de Longa Permanência, que atualmente abriga cerca de 37 idosos.

A amostra total foi constituída por 50 cuidadores ( 15 formais e 35 informais) e a análise do poder da amostra, apresentando como desfecho o desconforto emocional (escore total), empregando-se o aplicativo PASS (Power Analysis and Sample Size), versão de 2002, para um nível de significância de $\alpha=0,05$ e um tamanho amostral de $\mathrm{n}=50$, revelou um poder estatístico apriorístico de $1-\beta=66,4 \%$.

Foi considerado para os critérios de inclusão, cuidador informal, pessoa da família ou próxima do idoso, como um vizinho ou amigo que presta os cuidados, sem que haja qualquer tipo de contrato e pagamento para o fim de cuidar e o cuidador formal, considerando a pessoa maior 
de idade, com ensino fundamental e/ou médio completo, com treinamento específico em instituição oficialmente reconhecida para a atividade do cuidar e que recebe remuneração para a atividade, com ou sem vínculo à instituição. $\mathrm{O}$ critério de exclusão para os participantes era ter menos que 18 anos.

A coleta de dados foi realizada no primeiro semestre de 2014, por meio de entrevistas aos cuidadores. Após a aprovação do Comitê de Ética em Pesquisa da Universidade Federal de São Carlos, por meio da Plataforma Brasil, em 15/10/2013. Para a obtenção dos dados foi utilizado um instrumento contendo as seguintes variáveis e escalas:

Instrumento para caracterização do cuidador: permite conhecê-los nos aspectos: sexo, idade, estado conjugal, grau de parentesco, conhecimento sobre a doença, realização de curso formal, horas dedicadas ao cuidar, atividades do cuidar, se existe apoio e doenças auto-relatadas.

Escala de Sobrecarga de Zarit: elaborada por Zarit e Zarit ${ }^{7}$, traduzida e validada para a cultura brasileira por Scazufca ${ }^{8}$. Contém 22 itens, objetiva avaliar o impacto percebido do cuidar sobre a saúde física e emocional, atividades sociais e condição financeira. As respostas aos 22 itens devem ser dadas segundo uma escala de cinco pontos que descrevem como cada afirmação afeta a pessoa. O total da escala é obtido somando todos os itens e pode variar de 0 a 88 . Quanto maior a pontuação obtida, maior a sobrecarga percebida pelo cuidar. Foi utilizado ponto de corte, segundo estudo internacional de Ferreira et al. ${ }^{9}$, a seguinte pontuação: sobrecarga intensa, escores entre $61 \mathrm{e}$ 88 ; moderado a severo, entre 41 e 60 , moderado a leve, entre 21 e 40; e ausência de sobrecarga, escores inferiores a 21 pontos. Vale ressaltar que esta escala é validada para avaliação de cuidadores informais, dessa forma, para o presente estudo foi utilizada apenas a última questão da escala (O quanto se sente sobrecarregado?) para os cuidadores formais.

Self Reporting Questionnaire (SRQ-20):este instrumento, desenvolvido em 1980 por Harding et al. ${ }^{10} \mathrm{e}$ validado no Brasil em 1986 por Mari e Willians ${ }^{11}$, tem como objetivo a detecção de desconforto emocional na população geral. A pontuação pode variar de 0 a 20 e utilizado o ponto de corte de $7 / 8$ baseado no estudo brasileiro para diferenciar a possibilidade de casos com desordens emocionais.

A análise dos dados foi realizada no aplicativo Statistical Package for the Social Science (SPSS) versão 20.0, de forma descritiva e univariada, tan- to para variáveis categóricas (tabelas de frequência) quanto para variáveis quantitativas (medidas de tendência central e variabilidade). As médias das variáveis categóricas foram analisadas estatisticamente pelo Teste $\mathrm{T}$ de Student. Também realizou o cálculo de correlação de Pearson entre variáveis quantitativas. As correlações foram consideradas como sendo fracas $(r<0,3)$, moderadas $(0,3 \leq r<0,7)$ ou fortes $(r \geq 7)$. A análise bivariada incluiu medidas de associação em tabelas de contingência, para variáveis qualitativas, como qui-quadrado. Os postos médios das medidas de desconforto emocional (SRQ 20) foram analisados estatisticamente pelo teste não-paramétrico de Mann-Whitney com ajuste de Bonferroni. O nível de significância utilizado foi $\alpha=0,05$.

\section{Resultados}

A Tabela 1 apresenta o perfil dos 50 cuidadores entrevistados, sendo 15 formais avaliados na ILPI, e 35 informais, do Hospital Universitário (HU) e da Unidade Saúde Escola (USE). A amostra tem prevalência feminina tanto na amostra de cuidadores formais quanto na de informais. Ter companheiro e utilizar a religiosidade como suporte, foram mais relatados tanto pelos cuidadores formais quanto informais. Ressalta-se que o nível de escolaridade dos cuidadores formais é superior comparado aos cuidadores informais, sendo aqueles representados por pessoas com mais de 9 anos de escolaridade. Quanto a sobrecarga, nota-se que ambos, na maioria, se sentem um pouco sobrecarregados, porém os cuidadores informais também relatam muita $(25,7 \%)$ e extrema (14,3\%) sobrecarga, o desconforto emocional foi mais relatado pelos cuidadores informais $(45,7 \%)$, comparados aos formais $(26,7 \%)$. E por fim, as doenças autorreferidas, destacamse problemas de coluna para os cuidadores formais $(38,5 \%)$ e a Hipertensão Arterial Sistêmica $(45,7 \%)$ para os cuidadores informais.

Na Tabela 2, estão descritas as médias de idade, escolaridade, tempo de cuidado, horas diárias de trabalho, desconforto emocional e sobrecarga. Para os cuidadores informais, observa-se, idade mais avançada, menor escolaridade, mais tempo que exerce a função de cuidador, mais tempo em horas diárias de trabalho e maior frequência de desconforto emocional. Ressalta-se que para estes cuidadores foi possível aplicar a escala completa de sobrecarga (Zarit) obtendo-se o valor médio de 30,9 , o que corresponde a sobrecarga moderada a leve. 
Tabela 1. Distribuição das principais diferenças entre Cuidadores Formais e Informais, da ILPI e a somatória de cuidadores da USE e HU, São Carlos-SP, 2014.

\begin{tabular}{|c|c|c|c|c|}
\hline \multirow{2}{*}{ Perfil dos cuidadores } & \multicolumn{2}{|c|}{ ILPI } & \multicolumn{2}{|c|}{ USE/HU } \\
\hline & $\mathbf{N}^{\circ}$ & $(\%)$ & $\mathbf{N}^{\circ}$ & $(\%)$ \\
\hline \multicolumn{5}{|l|}{ Sexo } \\
\hline Feminino & 13 & 86,7 & 30 & 85,7 \\
\hline Masculino & 02 & 13,3 & 05 & 14,3 \\
\hline \multicolumn{5}{|l|}{ Escolaridade } \\
\hline 1 a 4 anos & - & - & 15 & 42,9 \\
\hline 5 a 8 anos & - & - & 6 & 17,1 \\
\hline 09 anos ou mais & 15 & 100 & 14 & 40 \\
\hline \multicolumn{5}{|l|}{ Tem companheiro? } \\
\hline Sim & 08 & 53,3 & 25 & 71,4 \\
\hline Não & 07 & 46,7 & 10 & 28,6 \\
\hline \multicolumn{5}{|l|}{ Suporte social (sim) } \\
\hline Instituição Religiosa & 10 & 66,7 & 08 & 22,9 \\
\hline Grupo apoio (saúde) & 01 & 6,7 & 04 & 11,4 \\
\hline \multicolumn{5}{|l|}{ Sobrecarga } \\
\hline Nem um pouco & 03 & 20 & 08 & 22,9 \\
\hline Um pouco & 06 & 40 & 11 & 31,4 \\
\hline Moderadamente & 04 & 26,7 & 02 & 5,7 \\
\hline Muito & 02 & 13,3 & 9 & 25,7 \\
\hline Extremamente & 0 & 0 & 5 & 14,3 \\
\hline \multicolumn{5}{|l|}{ Desconforto Emocional } \\
\hline Sim & 04 & 26,7 & 16 & 45,7 \\
\hline Não & 11 & 73,3 & 19 & 54,3 \\
\hline \multicolumn{5}{|l|}{ Doenças auto referidas } \\
\hline Hipertensão Arterial & 1 & 6,7 & 16 & 45,7 \\
\hline Diabetes & - & - & 10 & 28,6 \\
\hline Problemas na coluna & 6 & 38,5 & 10 & 28,6 \\
\hline Total & 15 & 100 & 35 & 100 \\
\hline
\end{tabular}

Tabela 2. Distribuição das médias de Idade, Escolaridade, Horas diárias de trabalho, Remuneração, Tempo Cuidador, Horas Semanais, Desconforto Emocional entre Cuidadores Formais da ILPI e a somatória de Cuidadores Informais da USE e HU. São Carlos-SP, 2014.

\begin{tabular}{lcccccc}
\hline \multirow{2}{*}{ Médias (desvio padrão) / Perfil Cuidador } & \multicolumn{3}{c}{ ILPI } & \multicolumn{3}{c}{ USE/HU } \\
\cline { 2 - 7 } & $\mathbf{M}(\mathbf{d p})$ & Mínimo & Máximo & M (dp) & Mínimo & Máximo \\
\hline Idade & $36,7(8,5)$ & 21 & 50 & $55,2(15,4)$ & 19 & 83 \\
Escolaridade & $13,7(2,9)$ & 11 & 23 & $7,1(3,9)$ & 1 & 13 \\
Horas diárias de trabalho & $7,3(2,6)$ & 5 & 12 & 19,8 & 6 & 24 \\
Tempo cuidador & $6,2(5,9)$ & 1 & 18 & $6,5(4,8)$ & 1 & 20 \\
Desconforto Emocional & $4,5(3,9)$ & 0 & 11 & $6,9(3,9)$ & 0 & 14 \\
Sobrecarga (ZARIT) & - & - & - & $30,9(14,3)$ & 5 & 63 \\
\hline
\end{tabular}

Depois de aplicada a correlação de Pearson, a prevalência de desconforto emocional para cada um dos dois tipos de cuidadores, não se encontrou associação entre o tipo de cuidador ou modalidade de cuidado e a proporção ou prevalência de desconforto emocional ( $r=0,208, p=0,345)$. O teste para postos de Mann-Whitney também não detectou uma diferença estatisticamente significativa $(\mathrm{p}=0,40)$ entre a média dos postos de sobrecarga para as duas modalidades de cuidado. 
Quanto aos aspectos relacionados à saúde dos cuidadores formais foi elencada a lombalgia $(38,5 \%)$ como sendo a principal. Para os cuidadores informais foram elencadas as doenças auto referidas, Hipertensão Arterial (40\%) seguida pela lombalgia (28,6\%). As demais (dislipidemia, Diabetes, cardiopatia, osteoporose, artrite/artrose, outras) foram pouco relatadas.

O desconforto emocional foi avaliado pelo SRQ 20, revelando que para o cuidador formal a questão "dormir mal" foi a mais relatada $(53,3 \%)$, seguida das questões "sentir-se tenso(a), nervoso(a) ou preocupado(a)" (40\%) e "sentirse cansado(a) (40\%). Para os cuidadores informais, a questão "sentir-se tenso(a), nervoso(a) ou preocupado(a)" foi a mais relatada (80\%), seguida das questões "sentir-se triste ultimamente" $(62,9 \%)$ e "dormir mal” (60\%).

Segundo as atividades que os cuidadores formais exercem relativas às Atividades de Vida Diária dos idosos, apenas a atividade física é pouco estimulada no contexto da ILPI, apenas 33,3\% dos cuidadores exercem com o idoso essa atividade. As demais atividades, principalmente no que se refere às ABVDs (higiene, eliminações, alimentação, medicação, sono/repouso) são executadas por $86,7 \%$ dos cuidadores. Dentre as ações mais realizadas pelos cuidadores informais estão: retorno às consultas $(97,1 \%)$, o auxílio com a medicação $(88,6 \%)$ e a alimentação $(68,6 \%)$. Em relação à atividade menos estimulada pelos cuidadores, destaca-se, também, a atividade física em um total de $80 \%$.

\section{Discussão}

Um dos pontos que chama a atenção na pesquisa é a prevalência do sexo feminino em ambas as amostras, de cuidadores formais e informais, fato já registrado na literatura ${ }^{12-15}$. Entender os motivos que levam uma mulher a se vincular a trabalhos dessa natureza e como ela se relaciona com ele, é fundamental para propor ações direcionadas e levando em consideração as especificidades de gênero.

Todos os cuidadores formais entrevistados relataram mais de nove anos de ensino, cursos e capacitações em relação ao cuidado do idoso, isso demonstra a preocupação da instituição em contratar profissionais qualificados e em oferecer um serviço pautado no que as legislações para ILPI's consideram, o que é uma característica bastante positiva. No entanto, é necessário alertar sobre a necessidade de educação permanente dessa equi- pe e de uma gestão participativa e multidisciplinar, que incentive o diálogo e faça com que esses profissionais sejam de fato considerados como parte fundamental dos processos terapêuticos ${ }^{16}$.

Quanto ao apoio recebido fora da instituição, os homens não relataram buscar ajuda, enquanto as mulheres citaram o apoio religioso como forma de suporte para o trabalho. A religião pode ser considerada um fator de motivação e suporte aos profissionais da saúde, oferecendo suporte aos enfermeiros na melhoria do serviço prestado. Além de se tratar de um elemento que pode ajudar na relação profissional-paciente ${ }^{17}$.

Com relação as queixas de saúde, os cuidadores apresentam comprometimentos de saúde e lesões diretamente ligadas ao seu processo de trabalho, como problemas na coluna, o que reporta a necessidade de se acompanhar o andamento desses casos, no sentido de se preservar a saúde funcional desses cuidadores. No presente estudo, cuidadores formais apresentaram maior prevalência de dores na coluna que cuidadores informais, sugerindo que não apenas a idade está relacionada aos sintomas físicos, mas também o desempenho da atividade de cuidador. Pinquart e Sörensen ${ }^{18}$, em uma meta-análise, verificaram que cuidadores de idosos possuem a saúde física em desvantagem quando comparados a grupos de não cuidadores.

Práticas de ginástica laboral, a adaptação da carga de trabalho e as características de saúde de cada cuidador podem ser incorporadas na tentativa de amenizar essas complicações. Contanto que a equipe se integre e busque soluções para isso, é um foco de atenção que tem um grande potencial para ser explorado, uma vez que a instituição já dispõe de profissionais de saúde e lazer, equipamentos e espaços para isso.

O estudo contribuiu também para o conhecimento sobre sintomas de sobrecarga experienciados pelos cuidadores de idosos que trabalham na ILPI, revelando o relato dos cuidadores entrevistados, com maior frequência, sentir-se "um pouco sobrecarregado" (40\%) e "moderadamente" $(26,7 \%)$. Em estudo com cuidadores de pacientes oncológicos, Maronesi et al. ${ }^{19}$ tiveram uma amostra de $18,7 \%$ da população estudada com sobrecarga, sendo uma porcentagem menor se comparada aos cuidadores informais (47\%), fator que se relaciona com os resultados encontrados nesta pesquisa.

Em outros estudos, como de Kappaun e Go$\mathrm{mez}^{20}$ e Meneghini et al. ${ }^{21}$, a sobrecarga foi uma das queixas mais relatadas pelos profissionais de enfermagem, por tratar-se de uma profissão que 
requer esforço físico frequentemente. Mesmo com dados menores de sobrecarga, é importante ficar atento aos sintomas de sobrecarga, já que muitas vezes os enfermeiros apresentam características da Síndrome de Burnout.

De maneira complementar à investigação sobre o sentimento de sobrecarga, avaliou-se problemas físicos e psicológicos que caracterizam o desconforto emocional do cuidador, em que $26,7 \%$ dos cuidadores foram diagnosticados com desconforto emocional, além do fato de relatarem, mais frequentemente, má qualidade de sono, sentimento de tensão e cansaço, dados próximos aos encontrados no estudo de Silva et al. ${ }^{22}$. Podemos relacionar isso, novamente, ao fato da jornada intensa de trabalho dos funcionários e ao fato de o número de idosos cuidados ser maior que dos cuidadores informais.

Esse relato nos mostra as especificidades do processo de trabalho em escalas de trabalho e também ao estresse a que são submetidos esses cuidadores, em que alguns sentimentos como preocupação e tensão nos cuidadores, e muitos aspectos podem estar envolvidos, como gravidade da doença do paciente, convivência diária e ininterrupta associada à prestação de cuidados, a dependência do mesmo e o despreparo dos cuidadores para tal enfrentamento, que também poderá repercutir na qualidade de sono dos mes$\operatorname{mos}^{23}$.

A falta de preparo dos cuidadores é de difícil solução em curto prazo, sendo necessário conhecer o perfil destes indivíduos, pois vivenciam problemas distintos, que estão relacionados às condições socioeconômico e culturais de cada família, para que seja prestada uma assistência mais direcionada, adequando as condutas à realidade de cada um e adaptando as orientações a cada tipo de cuidador e paciente.

Diante disso, Duarte ${ }^{24}$ buscou traçar diretrizes de uma política de atenção à pessoa idosa, elaborando um programa de capacitação global destinado a desenvolver mão de obra qualificada para assistir os idosos mais dependentes, segundo um eixo de orientação uniforme. Esse programa teve como objetivo, um "olhar gerontológico" aos profissionais que trabalham com idosos, além de orientação aos formadores de cuidadores de pessoas idosas e aos próprios cuidadores, tornandose referência em cursos de cuidadores de idosos no Brasil, o que pode ser considerado um marco para o desenvolvimento de ações voltadas aos cuidadores formais.

Sobre as características dos cuidadores informais, assim como na amostra de cuidadores for- mais, o sexo feminino teve prevalência, totalizando um total de $85,7 \%$ dos entrevistados. Ainda assim, 5 indivíduos do sexo masculino participaram da pesquisa, totalizando uma média de idade maior (61,8 anos), comparada ao sexo feminino (55,4 anos). Cardona et al..$^{25}$, em estudo parecido na cidade de Medellin, na Colômbia, apresentou resultados semelhantes, onde $81,6 \%$ da sua amostra era composta pelo sexo feminino, com média de idade de 56,5 anos e $73,7 \%$ declaravamse donas de casa como ocupação $0^{5,12}$.

A taxa mais alta de escolaridade variou de 1 a 4 anos de estudo, cerca de $42,9 \%$, seguida de mais de 9 anos de estudo (40\%). O resultado apresenta um fato curioso, onde é possível encontrar duas vertentes. $\mathrm{O}$ fato da diferença escolar pode ser relacionado à coleta ter sido realizada em um Hospital Escola e uma Unidade de Saúde Escola, onde ambos apresentam perfis institucionais diferentes, sendo que um apresenta um maior alcance da população da cidade e o outro apresenta um benefício de ingresso onde apenas alguns têm o privilégio de acesso.

Pereira et al. ${ }^{12}$, em estudo realizado na Unidade de Emergência do Hospital das Clínicas da Faculdade de Medicina de Ribeirão Preto (HCFMRP), apresentou população com escolaridade de 1 a 4 anos (33,9\%). Já em um estudo de Curitiba, $71 \%$ da amostra apresentaram mais que 8 anos de escolaridade ${ }^{15}$. Os hábitos de cultura e o contexto que ela está inserida ainda é um fator delimitante às oportunidades da população, fato que deve ser levado em consideração ao medir níveis de escolaridade.

A carga horária autorrelatada obteve uma média de 19 horas diárias para as mulheres e cerca de 11 horas para os homens, outro fator importante é a questão da média de tempo de cuidado ser maior para as mulheres (6,9 anos) quanto aos homens ( 3,8 anos). Ao deixar aberta a questão do número de horas, muitos idosos relatavam cuidar de outros durante 24 horas ao dia, quando questionados quanto ao tempo de dormir, muitos relatavam dormir mal por prestar o cuidado à noite. Tais médias foram maiores com aquelas encontradas no estudo de Gratão et al. ${ }^{2}$, com média de 15,6 horas/dia, referente a cuidadores de idosos demenciados, o que exige grande demanda. Divergente ao estudo de Pereira et al. ${ }^{12}$, em que se revelou média de 4,6 horas/dia de cuidado com idosos após AVE.

Quanto ao tempo de cuidado durante o dia em horas e quantos dias da semana, o cuidador assume que as tarefas do cuidado estão estreitamente interligados. Cuidadores que despendem 
maior tempo com os pacientes têm maior probabilidade de apresentar depressão e ansiedade ${ }^{26}$.

Muitas vezes, o sentimento de esgotamento ou exaustão dos cuidadores associado ao elenco de sintomatologia caracterizada no indivíduo que sofre de sobrecarga é resultante da grande dedicação e esforço na atividade de cuidar, na qual o cuidador desconsidera suas próprias necessidades, constituindo-se um problema psicossocial ${ }^{12}$.

Analisando as respostas na Escala de Zarit, nota-se a preocupação e a diferença que o peso quanto à relação familiar exerce na autopercepção do cuidador. Ao utilizar a última pergunta de base, de modo geral, 11 cuidadores declararam estar apenas um pouco sobrecarregado, enquanto 9 declararam estar muito sobrecarregado. Nota-se determinado receio dos cuidadores e sentimentos de culpa quando relatados casos de desconforto perante o cuidado do idoso. Ao avaliar 35 cuidadores na Colômbia, Ocampo et al. ${ }^{27}$ apresentou resultado parecido ao da pesquisa, onde, ao aplicar Zarit, 40\% apresentaram sobrecarga leve e 54,2\% não apresentaram sobrecarga.

No estudo de Seima et al. ${ }^{15}$, dos 208 cuidadores entrevistados, cerca de $26 \%$ apresentaram sobrecarga moderada a severa, se relacionando com os dados deste estudo onde $25,7 \%$ da amostra afirmaram se sentir muito sobrecarregado. Em relação ao score encontrado na pesquisa, 30,9 (sobrecarga moderada a leve), encontram-se outros estudos com valores próximos de sobrecarga como na pesquisa de Rodrigues et al. ${ }^{28}$ que obtiveram uma média de 26,5. Em geral, na maior parte dos estudos encontrados, os cuidadores familiares apresentavam grau de sobrecarga de leve a moderada, se relacionando com este estudo, além de apresentarem características semelhantes como gênero ${ }^{4,12,15,28}$.

Ao assumir a responsabilidade de cuidar, o cuidador informal limita-se a exercer funções que costumavam ser diárias em seu cotidiano e passa a voltar-se apenas às necessidades dos idosos, deixando de viver sua vida e interesses. Isso aumenta o nível de sobrecarga e desconforto emocional, ocasionando sentimentos negativos como solidão e isolamento social ${ }^{29}$.

Além da sobrecarga percebida, ao avaliar o desconforto emocional, cerca de $80 \%$ relatou se sentir nervoso e tenso, seguido de relato de sentimento de tristeza e queixas relacionadas à duração de sono, dados semelhantes encontrados no estudo de Morais et al. ${ }^{30}$, onde 44,3\% da amostra apresentou sinais de desgaste, sendo os mais relatados: sentir-se tenso ou preocupado $(73,8 \%)$, dormir mal $(63,9 \%)$ e sentimento de tristeza (55,7\%). Com relação ao SRQ 20, apenas o nível de sobrecarga obteve correlação significativa com o desconforto emocional $(\mathrm{p}<0,05)$, fato já relatado em outros estudos ${ }^{4}$.

Quando o cuidador é um familiar, a tarefa de cuidar pode ser percebida como mais desgastante, o que pode ocasionar inclusive situações conflitantes na família ${ }^{4}$.

Menos da metade da amostra apresentou desconforto emocional, porém foi possível notar que a questão da idade, escolaridade e carga horária durante a semana apresentaram relação com o desgaste emocional.

É necessário o conhecimento por meio de informações obtidas de instrumentos validados para que se possa implementar e avaliar ações que garantam melhor assistência a esses cuidadores e aos seus familiares idosos, considerando que os mesmos merecem atenção especial sob o aspecto psicológico.

\section{Conclusão}

Os dados revelaram que cuidadores informais apresentaram níveis de desconforto emocional, sentimentos de tristeza e horas diárias de trabalho (19,8 horas) maiores se comparados aos cuidadores formais, que demonstraram sentimentos de cansaço, má qualidade de sono e cumprimento de 7,3 horas diárias de trabalho. As consequências do cuidado informal podem estar relacionadas ao fato do cuidador ser uma pessoa próxima ou da família.

Dessa forma, ressalta-se a importância de profissionais mais capacitados para atender os idosos e a implementação de políticas públicas voltadas a essa população, que deveriam abordar diversos contextos, como planejamento de intervenções visando à melhoria nas condições de saúde e de trabalho destes indivíduos.

Apesar de uma amostra reduzida, o estudo foi de extrema relevância para caracterizar o perfil desses cuidadores e avaliar formas de minimizar e até mesmo evitar a sobrecarga e o estresse emocional. De acordo com a atual situação populacional do Brasil, o país necessita atentar-se às novas demandas relacionadas ao processo de envelhecimento de modo que este não se torne um problema para os profissionais e para os familiares.

O gerontólogo é um profissional chave para promover o cuidado integral à saúde do cuidador de idosos, o qual tem foco na otimização da qua- 
lidade de vida, por meio da transmissão de informações, com a sua competência, para formar uma equipe capaz de melhorar as condições dos serviços oferecidos, interferindo positivamente na resolutividade dos problemas dos cuidadores e idosos. Dessa forma, sugere-se aos serviços de geriatria e gerontologia planejar estratégias de suportes formais e informais, coordenadas por profissionais gerontólogos, para a atenção à saúde dos cuidadores de idosos.

Os resultados da presente pesquisa contribuem para o planejamento de uma sistematização de atenção individualizada respeitando cada perfil, privilegiando-se tarefas relacionadas à promoção de saúde, à prevenção de sobrecarga $\mathrm{e}$ desconforto emocional nos cuidadores de idosos.

\section{Colaboradores}

MAA Diniz, BRS Melo, KH Neri, FG Casemiro participaram da coleta de dados, elaboração do artigo, discussão, redação e revisão. CCLO Gaioli, LC Figueiredo e ACM Gratão participaram da análise de dados, correção e revisão. 


\section{Referências}

1. Instituto Brasileiro De Geografia E Estatística (IBGE). [homepage na internet]. Pesquisa Nacional por Amostra de Domicílios (PNAD, População). [acessado 2016 Abr 12]. Disponível em: http://www.ibge.gov.br/home/ estatistica/pesquisas/pesquisa_resultados.php?id_pesquisa $=40$

2. Gratão ACM, Vale FAC, Cruz MR, Haas VJ, Lange C, Talmelli, LFS, Rodrigues RA. Family caregivers demands from elderly individuals with dementia. Rev Esc Enferm USP. 2010; 4(4):873-880.

3. Couto AM, Castro EAB, Caldas CP. Vivências de ser cuidador familiar de idosos dependentes no ambiente domiciliar. Rev Rene 2016; 17(1):76-85.

4. Gratão ACM, Vendrúscolo TRP, Talmelli LFS, Figueiredo LC, Santos JLF, Rodrigues RAP. Sobrecarga e Desconforto Emocional em Cuidadores de Idosos. Texto contexto - enferm [Online]. 2012 abr-jun [acessado 2014 Oct 23] ; 21(2):304-312. Disponível em: http://www.scielo.br/scielo.php?pi$\mathrm{d}=$ S0104-07072012000200007\&script=sci_arttext

5. Stackfleth R, Diniz MA, Fhon JRS, Vendruscolo TRP, Fabricio-Whebe SCC, Marques S, Rodrigues RAP. Sobrecarga de trabalho em cuidadores de idosos fragilizados que vivem no domicílio. Acta Paul Enferm 2012. 25(3):768-774.

6. Olanda KKR, Passos XS, Dias CS. Perfil das morbidades dos cuidadores informais de idosos com Alzheimer. $J$ Health Sci 2015; 33(1):83-88.

7. Zarit SH, Zarit JM. The memory and behavior problems checklist: 1987R and the burden interview (technical report). Pennsylvania: University Park; 1987.

8. Scazufca M. Brazilian version of the Burden Interview Scale for the assessment of care in carers of people with mental illnesses. Rev Bras Psiquiatr 2002; 24(1):12-17.

9. Ferreira F, Pinto A, Laranjeira A, Pinto AC, Lopes A, Viana A, Rosa B, Esteves C, Pereira I, Nunes I, Miranda J, Fernandes P, Miguel S, Leal V, Fonseca C. Validação da escala de Zarit: sobrecarga do cuidador em cuidados paliativos domiciliários, para população portuguesa. Cadernos de Saúde [Online]. 2010; 3(2):13-19. [acessado 2015 Mar 29]. Disponível em: http://repositorio.ucp.pt/bitstream/10400.14/10936/1/CSaude_3-2_2010\%282\%29.pdf

10. Harding TW, Arango MV, Baltazar J. Mental disorders in primary health care: a study of their frequency and diagnosis in four developing countries. Psych Medicine 1980; 10(2):231-241.

11. Mari JJ, Williams P. A validity study of a psychiatric screening questionnaire (SRQ-20) in primary care in the city of São Paulo. Bras J Psychiatry 1986; 148:23-26.

12. Pereira RA, Santos EB, Fhon JRS, Marques S, Rodrigues RA. Sobrecarga dos cuidadores de idosos com acidente vascular cerebral. Rev Esc Enferm USP [Online]. 2013 [acessado 2014 Nov 05]; 47(1):185-192. Disponível em:

13. Loureiro LSN, Fernandes MGM, Marques S, Nóbrega MML, Rodrigues RAP. Sobrecarga de cuidadores familiares de idosos: prevalência e associação com características do idoso e do cuidador. Rev Esc Enferm USP [Online]. 2013 [acessado 2014 Nov 05]; 47 (5):11331140. Disponível em: http://www.scielo.br/pdf/reeusp/ v47n5/pt_0080-6234-reeusp-47-05-1129.pdf
14. Wachholz PA, Santos RCC, Wolf LSP. Reconhecendo a sobrecarga e a qualidade de vida de cuidadores familiares de idosos frágeis. Rev Bras Geriatr Gerontol [Online]. 2013 [acessado 2014 Nov 05]; 13(3):513-526. Disponível em: http://www.scielo.br/pdf/rbgg/v16n3/ v16n3a10.pdf

15. Seima MD, Lenardt MH, Caldas CP. Relação no cuidado entre o cuidador familiar e o idoso com Alzheimer. Rev Bras Enf [Online]. 2014 [acessado 2014 Nov 06]; 67(2). Disponível em: http://www.scielo.br/scielo.php?scrip$\mathrm{t}=$ sci_arttext\&pid=S0034-71672014000200233

16. Santos SSC, Feliciani AM, Silva BT. Perfil de idosos residentes em instituição de longa permanência: proposta de ações de enfermagem/saúde. Rev RENE. 2012; $8(3): 26-33$.

17. Teixeira JJV, Lefèvre F. Religiosidade no trabalho das enfermeiras da área oncológica: significado na ótica do discurso do sujeito coletivo. Rev. Bras. Cancerol [Online]. 2007. [acessado 2014 Nov 24]; 53(2):159-166. Disponível em: http://wwwl.inca.gov.br/rbc/n_53/v02/ pdf/artigo2.pdf

18. Pinquart M, Sörensen S. Differences between caregivers and noncaregivers in psychological health and physical health: a meta-analysis. Psychol Aging. 2003. [acessado $2015 \mathrm{Fev} 24$ ]; 18(2):250-267. Disponível em: http://psycnet.apa.org/journals/pag/18/2/250.pdf

19. Maronesi LC, Silva NR, Cantu SO, Santos AR. Indicadores de estresse e sobrecarga em cuidadores formais e informais de pacientes oncológicos. Estud. Pesqui. Psicol. 2014; 14(3):877-892.

20. Kappaun NR, Gomez CM. O trabalho de cuidar de pacientes terminais com câncer. Cien Saude Colet 2013; 18(9):2549-2557.

21. Meneghini F, Paz AA, Lautert L. Fatores ocupacionais associados aos componentes da síndrome de Burnout em trabalhadores de enfermagem. Texto contexto enferm [Online]. 2011 June. [acessado 2014 Nov 23]; 20(2):225-33. Disponível em: http://www.scielo.br/ $\mathrm{pdf} / \mathrm{tce} / \mathrm{v} 20 \mathrm{n} 2 / \mathrm{a} 02 \mathrm{v} 20 \mathrm{n} 2$

22. Silva JLL, Melo ECP, Griep RH, Rotemberg L. Estresse, trabalho e ambiente: prevalência de transtornos mentais comuns entre trabalhadores de enfermagem de um hospital federal no município do Rio de Janeiro. In: Semana Científica da Escola de Enfermagem Aurora de Afonso Costa, 15. 2008. [acessado 2015 Mar 30]. Anais eletrônicos. Niterói: UFF, 2008. Disponível em: www. uff.br/anaissegerenf/premio/texto\%20uff.semana.pdf

23. Cordeiro L, Albuquerque C. Cuidador informal de idosos dependentes: dificuldades e sobrecarga. [dissertação]. Viseu: Instituto Politécnico de Viseu, 2011.

24. Duarte YA. Manual dos formadores de cuidadores de pessoas idosas. São Paulo: Secretaria Estadual de Assistência e Desenvolvimento Social, Fundação Padre Anchieta: 2009.

25. Cardona D, Segura A, Berberí D, Agudelo M. Prevalencia y factores asociados al síndrome de sobrecarga del cuidador primario de ancianos. Rev. Fac. Nac. Salud Pública [Online]. 2013. [acessado 2014 Nov 05]; 31(1):30-39. Disponível em: http://www. scielo.org.co/scielo.php?script=sci_arttext \&pid=S0120-386X2013000100004 
26. Cassis SVA, Karnakis T, Moraes TA, Curiati JAE, Quadrante ACR, Magaldi RM. Correlação entre o estresse do cuidador e as características clínicas do paciente portador de demência. Rev Assoc Med Bras 2007; 53(6):497-501.

27. Ocampo JM, Herrera JA, Torres P, Rodriguez JA, Loboa L, Garcia CA. Sobrecarga asociada com el cuidado de ancianos dependientes. Colomb Med [Online]. 2007. [acessado 2014 Nov 05]; 38(1):40-46. Disponível em: http://bibliotecadigital.univalle.edu.co/bitstream/ 10893/4702/1/Caregiver.pdf

28. Rodrigues JEG, Machado ALG, Vieira NFC, Fernandes AFC, Rebouças CBA. Qualidade de vida e sobrecarga de cuidadores familiares de idosos dependentes. Cienc. Enferm. 2014; 20(3):119-129.

29. Lopes LO, Cachioni M. Intervenções psicoeducacionais para cuidadores de idosos com demência: uma revisão sistemática. J Bras Psiquiatr 2012; 61(4):252-261.

30. Morais HCC, Soares AMG, Oliveira ARS, Carvalho CML, Silva MJ, Araujo TL. Sobrecarga e modificações de vida na perspectiva dos cuidadores de pacientes com acidente vascular cerebral. Rev. Latino-Am. Enfermagem 2012; 20(5):944-953.

Artigo apresentado em 14/03/2016

Aprovado em 28/02/2017

Versão final apresentada em 02/03/2017 